\title{
Anatomic reconstruction for acromioclavicular joint injuries: a pilot study of a cost-effective new technique
}

\author{
Radhakrishnan Pattu, Girinivasan Chellamuthu, Kumar Sellappan, Chendrayan Kamalanathan \\ Department of Orthopedics, Government Mohan Kumaramangalam Medical College Hospital, Salem, India
}

\begin{abstract}
Background: The treatment for acromioclavicular joint injuries (ACJI) ranges from a conservative approach to extensive surgical reconstruction, and the decision on how to manage these injuries depends on the grade of acromioclavicular (AC) joint separation, resources, and skill availability. After a thorough review of the literature, the researchers adopted a simple cost-effective technique of AC joint reconstruction for acute ACJI requiring surgery.

Methods: This was a prospective single-center study conducted between April 2017 and April 2018. For patients with acute ACJI more than Rockwood grade 3, the researchers performed open coracoclavicular ligament reconstruction using synthetic sutures along with an Endobutton and a figure of 8 button plate. This was followed by AC ligament repair augmenting it with temporary percutaneous AC K-wires. Clinical outcomes were evaluated using the Constant Murley shoulder score.

Results: Seventeen patients underwent surgery. The immediate postoperative radiograph showed an anatomical reduction of the AC joint dislocation in all patients. During follow-up, one patient developed subluxation but was asymptomatic. The mean follow-up period was 30 months (range, 24-35 months). The mean Constant score at 24 months was 95. No AC joint degeneration was noted in follow-up X-rays. The follow-up X-rays showed significant infra-clavicular calcification in 11 of the 17 patients, which was an evidence of a healed coracoclavicular ligament post-surgery.

Conclusions: This study presents a simple cost-effective technique with a short learning curve for anatomic reconstruction of acute ACJI.

The preliminary results have been very encouraging.
\end{abstract}

Keywords: Acromioclavicular joint; Coracoclavicular reconstruction; Open technique; Anatomical reconstruction; Shoulder

\section{INTRODUCTION}

Acromioclavicular joint injuries (ACJI) are commonly seen in orthopedic surgery and sports medicine [1]. They often occur in athletes and youth after blunt force to the shoulder. The true incidence of ACJI has not been accurately reported, as many minor cases are missed [1]. The injured AC joint can cause persistent pain and compromise the function of the shoulder joint. The treatment of ACJI is controversial and ranges from a conservative approach to extensive surgical reconstruction. The decision on the best way to manage these dislocations depends on the grade of AC joint separation, availability of appropriate skills, and resources.

A wide variety of operative techniques using K-wires, hook

Received: May 23, $2021 \quad$ Revised: July 5, $2021 \quad$ Accepted: July 11, 2021

Correspondence to: Radhakrishnan Pattu

Department of Orthopedics, Government Mohan Kumaramangalam Medical College Hospital, Salem, Tamil Nadu 636002, India

Tel: +91-9443048299, E-mail: arthrork7@gmail.com, ORCID: https://orcid.org/0000-0002-0526-1117

Financial support: None.

Conflict of interest: None.

Copyright@ 2021 Korean Shoulder and Elbow Society.

This is an Open Access article distributed under the terms of the Creative Commons Attribution Non-Commercial License (http://creativecommons.org/licenses/by-nc/4.0/) which permits unrestricted non-commercial use, distribution, and reproduction in any medium, provided the original work is properly cited. 
plates, button plates, suture anchor fixation, and ligament reconstruction have been reported with different success rates [2,3]. After a thorough review of the literature, the authors decided to adopt a simple, cost-effective technique of AC joint reconstruction for acute ACJI requiring surgery. They performed coracoclavicular (CC) ligament reconstruction using synthetic sutures and AC ligament repair augmenting it with temporary percutaneous AC K-wires.

In this study, the authors intended to detail their technique, explain the rationale and merits behind such a technique, and report on the functional outcomes with a 2 -year follow-up. To their best knowledge, such a technique has not yet been reported in the literature.

\section{METHODS}

This was a prospective single-center study conducted between April 2017 and April 2018. The approval of Institutional Ethical Committee of Government Mohan Kumaramangalam Medical College Hospital was obtained (No. 76/LE/18221). Detailed consent was obtained from the patients. The study excluded patients with chronic ACJI (of more than 2 weeks duration), Acute ACJI-Rockwood grade $\leq$ III, previous history of clavicle and acromion fractures, patients more than 60 years of age or less than 20 years of age, and those with a history of chronic shoulder pain. Diagnosis and grading of AC injury was conducted based on a standard anteroposterior stress radiograph of the involved shoulder. The radiological examination included anteroposterior, axillary, and Zanca radiographic views [4]. Clinical outcomes were evaluated using the Constant score [5].

\section{Operative Technique}

Under general anesthesia, the patient was placed in the supine position with a sandbag under the ipsilateral scapula. The shoulder and upper extremity were prepped and draped. A 5-cm sabercut incision was made along the Langer's line, starting $1 \mathrm{~cm}$ posterior to the clavicle and extending anterior to the clavicle (Fig. 1), $2.5 \mathrm{~cm}$ medial to the AC joint. The deltoid and trapezius muscles were elevated subperiosteally from the distal clavicle and anterior acromion.

The base of the coracoid was prepared. The medial and the lateral ends of the coracoid were visualized. The center of the coracoid was marked. The entire length of the coracoid was then drilled with a 4-mm endoscopic reamer directing it slightly anteriorly, respecting neurovascular structures below. The titanium endo button (Onbutton; Biotek, Vadodara, Gujarat, India) was loaded with three size-2 fiber wires (BioFiber; Biotek, Vadodara,
Gujarat, India) manually and was pushed through the coracoid using a 3.2-mm plunger. The Endobutton flipped under the coracoid (Fig. 2). A 3.2-mm drill bit was used to create two tunnels through the superior cortex of the clavicle over the footprint of the two parts of the CC ligament-the trapezoid and the conoid respectively-about $1 \mathrm{~cm}$ apart and 2 to $5 \mathrm{~cm}$ from the lateral end of the clavicle, one anterolaterally and the other posteromedially. A large, curved cutting needle was passed through these tunnels to retrieve three strands of the fiber wire through each hole. The strands were then passed through the two holes of an eight plate or a two-holed reconstruction plate (Universal Co., Salem, India) placed over the top of the clavicle. The AC joint was reduced to a small degree below its normal anatomical position, and the ends of fiber wires were tied and tightened over the plate (Fig. 3). The AC ligaments were now repaired if both the ends of torn ligaments were identifiable. If not, the acromial part was tied to the button plate using size 2 ethibond (Fig. 3). Then, 1.8-mm K-wires were passed across the AC joint from outside without exploring the joint to augment the repaired AC ligaments. The delto-trapezial aponeurosis and fascia were meticu-

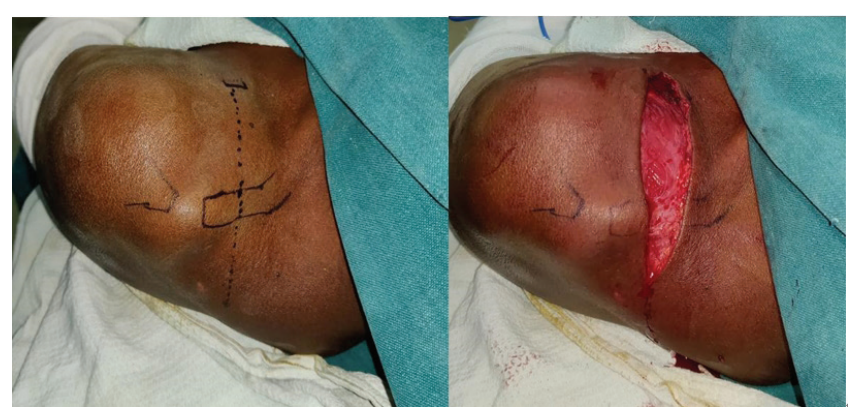

Fig. 1. Sabercut incision.

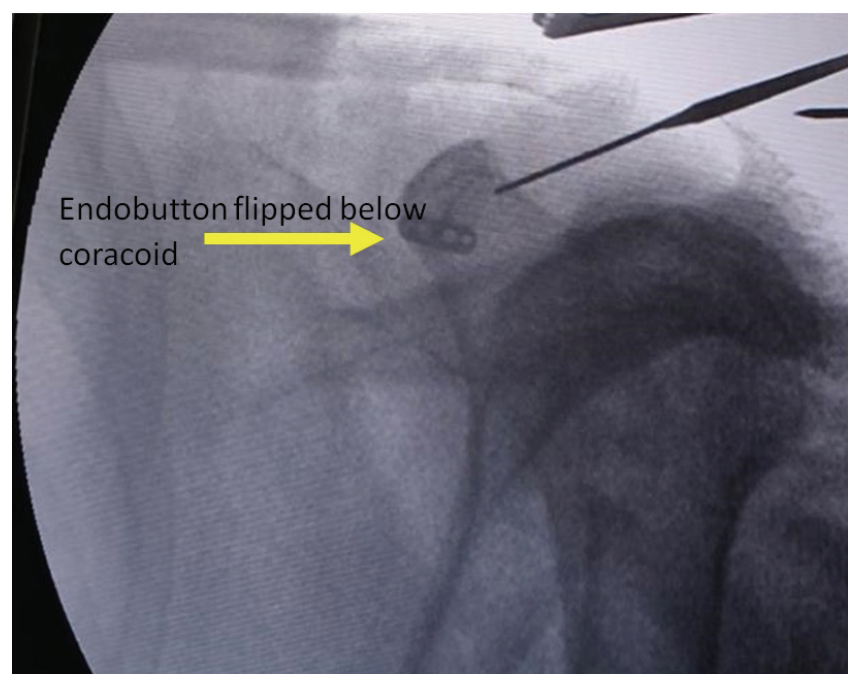

Fig. 2. C arm image after flipping the Endobutton. 
lously closed. Skin closure was performed. The important steps of the surgical technique are illustrated in the form of a line diagram in Fig. 4.

Postoperatively, the shoulder was protected in a sling for 3 weeks. Pendulum exercises were started 2 weeks after surgery. After the third week, K-wires were removed and the patients

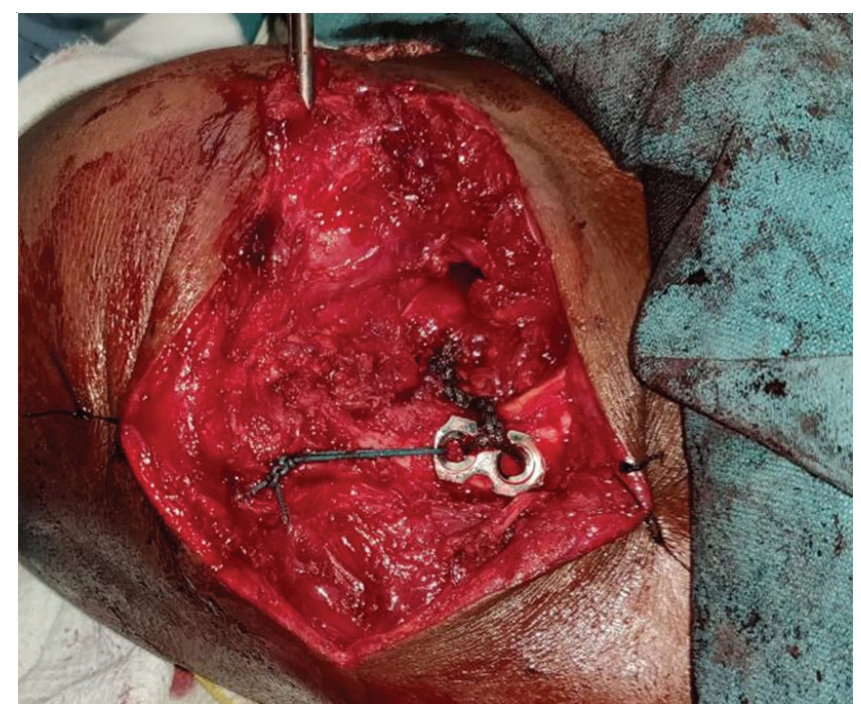

Fig. 3. Acromioclavicular ligament being repaired to the 8 plate. Note the oblique position of the plate indicating an anterolateral and a posteromedial position of drill holes on the clavicle, replicating the natural anatomy. were taught progressive range of motion exercises. Heavy weightlifting and resistance exercises were allowed after 3 months of surgery.

\section{RESULTS}

Seventeen patients ( 4 females and 13 males) with acute ACJI who met study criteria received surgical intervention and were followed for an average of 2 years. The average age of presentation was 37 years (standard deviation [SD], 7.6). Average time of presentation since injury was 4.6 days (SD, 2.6). All cases were Rockwood grade 5 injuries. The immediate postoperative radiograph showed an anatomical reduction of the AC joint dislocation in all patients (Fig. 3). No neurovascular complications were noted postoperatively. No implant-related and soft tissue complications were encountered.

During follow-up, one patient developed subluxation but was asymptomatic. The mean follow-up period was 30 months (range, 24-35 months). All patients had a clinically good range of movements and pain-free joints by an average of 10 weeks post-procedure. The mean Constant score at 24 months was 95 (SD, 3.5). No AC joint degeneration was noted in follow-up $\mathrm{X}$-rays (Fig. 5). Follow-up x-rays showed significant infra-clavicular calcification (Fig. 6) in 11 of the 17 patients, which is an evidence of a healed CC ligament [6].
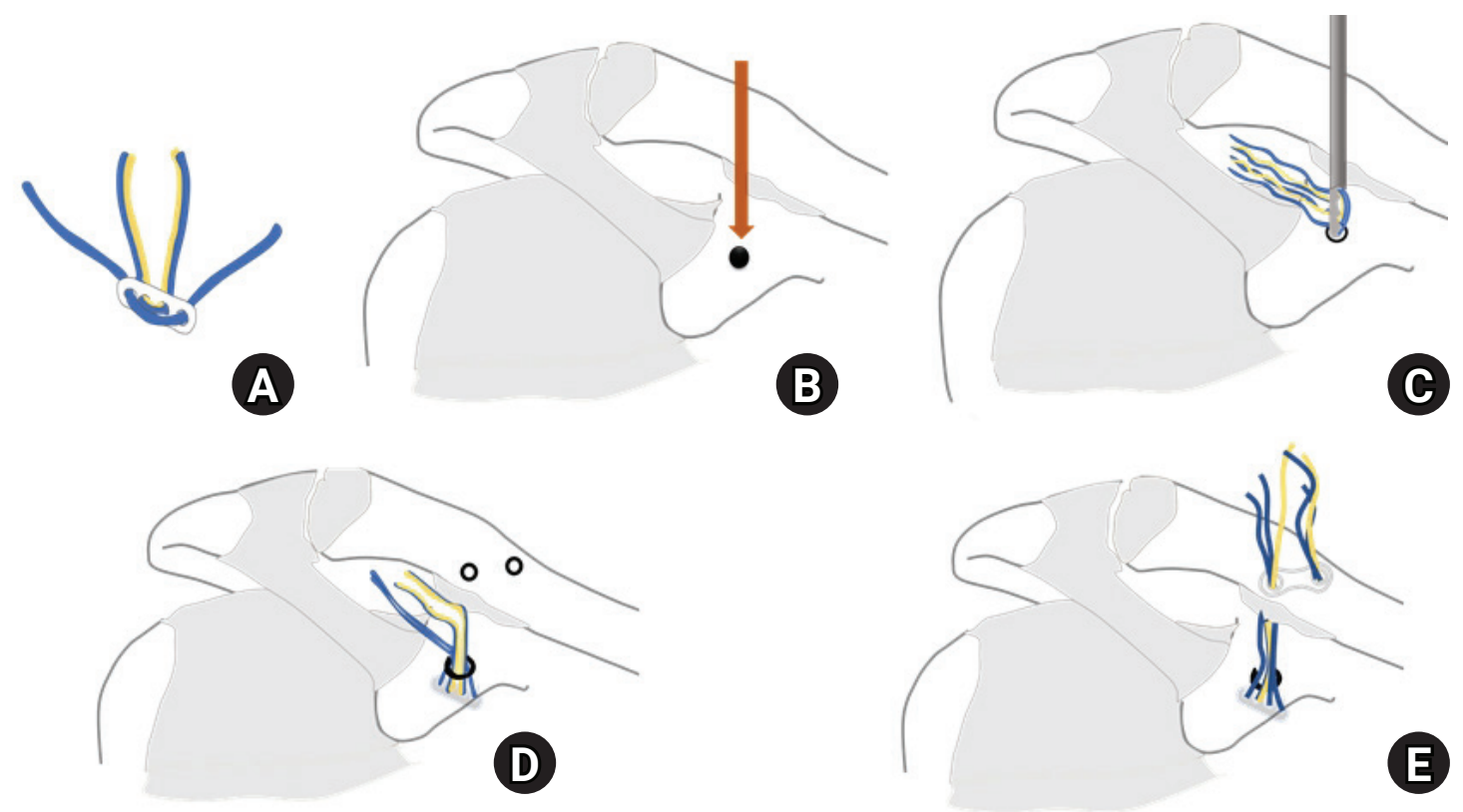

Fig. 4. Line diagram illustrating important steps of surgery. (A) Free Endobutton mounted on 3 fiber wires. (B) Point marked on the center of the coracoid near its base for coracoid tunnel. (C) Using 3.2-mm plunger the Endobutton is passed through the tunnel. (D) Endobutton is flipped below the coracoid. Drill holes over the clavicle are marked over the conoid and trapezoid footprints. (E) Fiber wires shuttled over the clavicle over the 8 plate. 

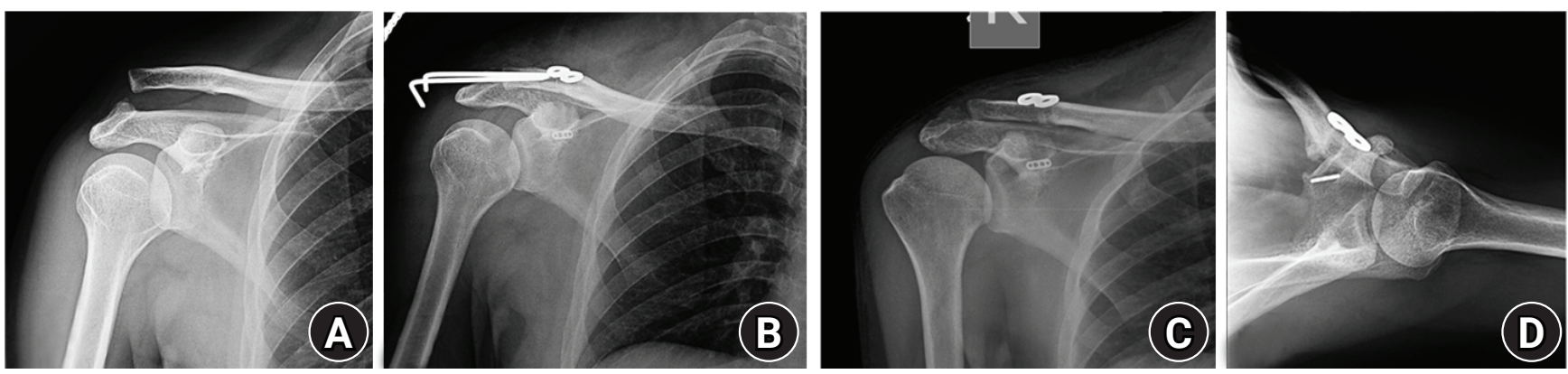

Fig. 5. Case example. (A) Preoperative X-ray showing type 5 acromioclavicular joint disruption. (B) Immediate postoperative X-ray with K-wires (C) in situ and (D) follow-up anteroposterior and axillary views after 25 months.

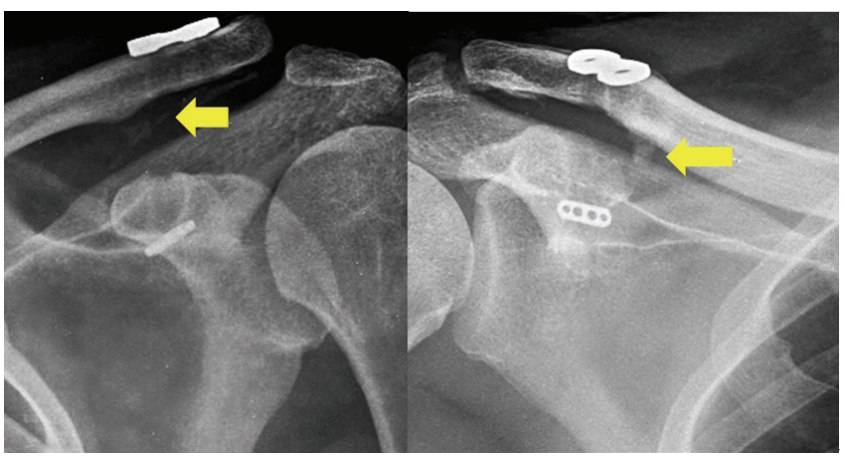

Fig. 6. Infraclavicular calcifications (arrows) in the follow-up X-rays of the patients.

\section{DISCUSSION}

Understanding the pathoanatomy and mechanism of injury is important for adopting an appropriate surgical technique. The joint capsule or AC ligament (i.e., thickenings in the capsule) and the extracapsular CC ligaments provide static stability to the AC joint $[7,8]$. Physiologic forces and the weight of the arms place significant translational forces in the vertical, anteroposterior, and axial planes of the $\mathrm{AC}$ joint. AC ligaments are the primary restraints of anterior translation [8]. The CC ligaments are formed by the conoid medially and the trapezoid laterally. They are the primary restraint to the inferior and medial translation of the scapulohumeral complex in relation to the clavicle [9]. The conoid ligament is attached proximally on the posteromedial aspect under the surface of the clavicle, typically $4.5 \mathrm{~cm}$ from the AC joint (47.2 $\mathrm{mm}$ in men and $42.8 \mathrm{~mm}$ in women) [7]. It tenses under loads that force the clavicle superiorly (or the scapula inferiorly). The trapezoid attaches proximally to the anterolateral aspect of the inferior clavicle, approximately $2.5 \mathrm{~cm}$ from the joint (25.4 $\mathrm{mm}$ in men and $22.9 \mathrm{~mm}$ in women) [8]. It tenses when there is medialization of the scapulohumeral complex, i.e., compression of the AC joint. The delto-trapezial aponeurosis and fascia provide dynamic stabilization to the AC joint, especially the anterolateral deltoid insertion [10].

The typical trauma mechanism is a force that depresses the shoulder girdle, such as that occurring in a fall from a two-wheeler on the shoulder or during a collision in contact sports. The force depresses the scapulohumeral complex resulting in tears of the AC and the CC ligaments [10]. The authors used the Rockwood classification [11] to grade ACJI. Surgery is generally indicated for types 4, 5, and 6 injuries. Conservative treatment is advised for types 1 and 2 . Type 3 injuries are initially treated conservatively. Surgical intervention is done if needed later on [1214].

Numerous surgical repair or reconstruction techniques have been published. In 2013, the number of different surgical techniques described was 162 [15]. Surgical procedures have gradually evolved from hardware fixation of the AC and CC joints through the reconstruction of ligaments by an array of methods such as utilization of the coracoacromial ligament, use of autologous or allografts, suture anchors, and flipped buttons to miniopen, arthroscopy assisted, and all arthroscopic techniques [16].

With increased understanding of the biomechanics of the AC joint, there is a greater inclination towards the use of anatomic ligament reconstruction techniques such as the use of synthetic sutures in the form of anchors or buttons, synthetic tapes, or auto or allografts, as they help in regaining pre-trauma biomechanics and function of the joint [15].

Beitzel et al. [15], in their systematic review, have described anatomic reconstruction as "the one that respects the bony anatomy of the clavicle and the acromion and reconstructs both the conoid and the trapezoid ligaments respecting their anatomy and individual function." They emphasized that the reconstruction should allow the complex three-dimensional motion pattern of the shoulder joint complex without compromising the stability of the construct. They preferred reinforcement of AC ligaments in the form of reefing of the overlying fascia. 
Among the various methods of anatomic reconstructions, use of synthetic suture materials is one of the simplest methods available. Walz et al. [6], in a cadaveric study of 40 shoulders, did a load test on native and reconstructed AC joints. They used two tight ropes for the reconstruction of CC ligaments and concluded that the technique is a stable and functional anatomic reconstruction procedure. In their cadaver study of Jerosch et al. [17] also emphasized a similar model. Synthetic suture reconstruction is recommended for acute injuries ( $<2$ weeks) [15] as there is a high chance of healing natural ligaments post-reconstruction. In chronic injuries, it is recommended to use grafts for ligament reconstruction as chances of healing are minimal [15].

Though studies support the reconstruction of the CC ligament alone, it does not restore the complete natural anatomy as the additional anteroposterior stability provided by the AC ligaments is lacking $[8,18]$. Lädermann et al. [19] used nonabsorbable sutures in the form of cerclage wires to reconstruct AC ligaments. Jerosch et al. [17] pointed out that anterior dislocation of the clavicle may result after some forms of CC reconstruction. Reconstruction of the AC ligament not only provides AP stability but also reduces the stress on the reconstructed CC ligament. Persistent AP instability has been described as a cause of chronic shoulder pain after AC joint reconstruction [19].

The authors have addressed acute ACJI considering all the inputs from the literature. Respecting the natural anatomy of conoid and trapezoid, they used synthetic sutures to reconstruct the ligaments. They restored anteroposterior stability by repairing the AC ligaments, closing the delto-trapezial aponeurosis and fascia, and temporarily augmenting this with $\mathrm{K}$-wires until the ligaments healed. The load to failure of CC ligaments was $500 \pm 134 \mathrm{~N}$, which was much less when compared to the material properties of the synthetic sutures that they have used $[8,20]$. The appearance of infraclavicular calcifications show healed CC ligaments which will provide permanent AC joint stability [6].

The advantages of this procedure include that this is a simple cost-effective procedure with a short learning curve when compared to similar all-arthroscopic and arthroscopy-assisted procedures. The authors used fiber wires, a free endo button, and button plates. This significantly brought down the cost when compared to implants such as suture anchors or sutures tapes and allografts. The other advantage compared to the arthroscopic technique is the ability to repair AC ligament and delto-trapezial aponeurosis and fascia.

However, this was a pilot study with a small sample size. Since significant complications were not experienced, further randomized controlled trials involving this technique will provide guidance in the right direction.
The authors have presented a simple cost-effective technique with a short learning curve for anatomic reconstruction of acute ACJI. Preliminary results have been very encouraging. Further large studies are needed to validate the technique.

\section{ORCID}

Radhakrishnan Pattu https://orcid.org/0000-0002-0526-1117

Girinivasan Chellamuthu https://orcid.org/0000-0001-5800-714X

Kumar Sellappan https://orcid.org/0000-0002-7094-5005

Chendrayan Kamalanathan https://orcid.org/0000-0003-4250-6379

\section{REFERENCES}

1. Chillemi C, Franceschini V, Dei Giudici L, et al. Epidemiology of isolated acromioclavicular joint dislocation. Emerg Med Int 2013;2013:171609.

2. Folwaczny EK, Yakisan D, Stürmer KM. The Balser plate with ligament suture: a dependable method of stabilizing the acromioclavicular joint. Unfallchirurg 2000;103:731-40.

3. Faraj AA, Ketzer B. The use of a hook-plate in the management of acromioclavicular injuries. Report of ten cases. Acta Orthop Belg 2001;67:448-51.

4. Zanca P. Shoulder pain: involvement of the acromioclavicular joint. (Analysis of 1,000 cases). Am J Roentgenol Radium Ther Nucl Med 1971;112:493-506.

5. Constant CR, Murley AH. A clinical method of functional assessment of the shoulder. Clin Orthop Relat Res 1987;(214): 160-4.

6. Walz L, Salzmann GM, Fabbro T, Eichhorn S, Imhoff AB. The anatomic reconstruction of acromioclavicular joint dislocations using 2 TightRope devices: a biomechanical study. Am J Sports Med 2008;36:2398-406.

7. Rios CG, Arciero RA, Mazzocca AD. Anatomy of the clavicle and coracoid process for reconstruction of the coracoclavicular ligaments. Am J Sports Med 2007;35:811-7.

8. Willimon SC, Gaskill TR, Millett PJ. Acromioclavicular joint injuries: anatomy, diagnosis, and treatment. Phys Sportsmed 2011;39:116-22.

9. Mazzocca AD, Spang JT, Rodriguez RR, et al. Biomechanical and radiographic analysis of partial coracoclavicular ligament injuries. Am J Sports Med 2008;36:1397-402.

10. Reid D, Polson K, Johnson L. Acromioclavicular joint separations grades I-III: a review of the literature and development of best practice guidelines. Sports Med 2012;42:681-96.

11. Gorbaty JD, Hsu JE, Gee AO. Classifications in brief: Rockwood classification of acromioclavicular joint separations. Clin Or- 
thop Relat Res 2017;475:283-7.

12. Galasso O, Tarducci L, De Benedetto M, et al. Modified Weaver-Dunn procedure for type 3 acromioclavicular joint dislocation: functional and radiological outcomes. Orthop J Sports Med 2020;8:2325967120905022.

13. Modi CS, Beazley J, Zywiel MG, Lawrence TM, Veillette CJ. Controversies relating to the management of acromioclavicular joint dislocations. Bone Joint J 2013;95:1595-602.

14. Korsten K, Gunning AC, Leenen LP. Operative or conservative treatment in patients with Rockwood type III acromioclavicular dislocation: a systematic review and update of current literature. Int Orthop 2014;38:831-8.

15. Beitzel K, Cote MP, Apostolakos J, et al. Current concepts in the treatment of acromioclavicular joint dislocations. Arthroscopy 2013;29:387-97.

16. van Bergen CJ, van Bemmel AF, Alta TD, van Noort A. New in- sights in the treatment of acromioclavicular separation. World J Orthop 2017;8:861-73.

17. Jerosch J, Filler T, Peuker E, Greig M, Siewering U. Which stabilization technique corrects anatomy best in patients with AC-separation? An experimental study. Knee Surg Sports Traumatol Arthrosc 1999;7:365-72.

18. Breslow MJ, Jazrawi LM, Bernstein AD, Kummer FJ, Rokito AS. Treatment of acromioclavicular joint separation: suture or suture anchors. J Shoulder Elbow Surg 2002;11:225-9.

19. Lädermann A, Grosclaude M, Lübbeke A, et al. Acromioclavicular and coracoclavicular cerclage reconstruction for acute acromioclavicular joint dislocations. J Shoulder Elbow Surg 2011; 20:401-8.

20. Najibi S, Banglmeier R, Matta J, Tannast M. Material properties of common suture materials in orthopaedic surgery. Iowa Orthop J 2010;30:84-8. 